\title{
Birth Brachial Plexus Palsy Update
}

\author{
Maureen R. Nelson · Arturo H. Armenta
}

Published online: 10 April 2014

(C) Springer Science + Business Media New York 2014

\begin{abstract}
Brachial plexus palsy evaluation and treatment have grown over the past few decades. In this review article we will address advances in evaluation and treatment, both surgically and conservatively, as well as new findings in outcomes, function and theories on central nervous system involvement. We will also touch on scientific impact other than from the field of medicine.
\end{abstract}

Keywords Birth brachial plexus palsy · Electrodiagnosis . Electrical stimulation $\cdot$ Nerve injury $\cdot$ Nerve graft

\section{Introduction}

Management of birth brachial plexus palsy (BBPP) has evolved dramatically over the past 20 years. There have been advances in evaluation and treatment, surgical and otherwise. Improvements in general scientific knowledge have contributed to this on an anatomical and physiological basis, perhaps most vividly manifest by improved imaging. Sciences outside of medicine have also contributed with mathematical and engineering models of forces, joints and muscles adding to progress in treatment. Research on

M. R. Nelson $(\square)$

Dell Children's Medical Center, Pediatric Subspecialty Services, 1301 Barbara Jordan Blvd., Suite 200, Austin, TX 78723, USA

e-mail: mrnelson@seton.org

\section{A. H. Armenta}

The Texas Brachial Plexus Institute, 6560 Fannin St., Suite 1804, Houston, TX 77030, USA

e-mail: art@drarmenta.com function and outcomes in this field is in early stages of development.

\section{Evaluation}

Evaluations are done with clinical examination, electrodiagnosis and imaging. Decisions regarding surgical intervention have been classically made at approximately 3 months of age. A recent study though showed that the best predictor of a severe BBPP was active elbow extension and flexion and electromyography (EMG) of the biceps at 1 month of age [1]. Another study of 48 infants with BBPP supports this, demonstrating that EMG at 1 month of age was more sensitive than those at 1 week or 3 months. Absent motor unit potentials at 1 month correlated with future absence of clinical elbow flexion [2•]. Another small study showed all children who regained elbow flexion by 1 month had normal shoulder function, without functional limitations, but those who regained elbow flexion after 3 months had restricted shoulder movement and less efficient use of the arm, as well as difficulty with ADLs [3]. A comparison study indicated that electrodiagnostic studies were more sensitive than CT myelogram for detecting a postganglionic rupture, but the reverse was true for preganglionic root avulsions [4]. EMG is also useful in evaluating older children with BPP. Infraspinatus muscle recruitment on EMG correlates with active shoulder external rotation as well as muscle volume on MRI [5]. Surface EMG on the long and short heads of the biceps during upper extremity tasks and compared to the contralateral limb showed EMG activity duration was higher in the long head of the biceps during overhead tasks in the affected arm. It was theorized that overactivity of this long head of the biceps may contribute to development 
of elbow flexion contracture as opposed to a more classic hypothesis of elbow flexor/extensor muscle imbalance [6॰]. An intraoperative study of eight patients who had fascicle transfers showed that those who had a donor fascicle selected without electrodiagnosis had poorer recovery than those who had quantitative electrodiagnosis guiding the selection of the donor fascicles [7].

Other recent studies of clinical distinctions in evaluation include the finding that clinically active elbow extension at 1 month is a significant predictor of a C5-6 lesion [1] and that Horner's syndrome is not proof of a preganglionic C8T1 lesion [8]. Decreased glenohumeral portion of shoulder movement and increased scapulothoracic contribution to movement in overhead shoulder movements have been described in children with upper plexus palsy [9].

Biomechanical models are now being used to evaluate joint forces in children with BBPP. Healthy children have up to $10^{\circ}$ of internal rotation of the shoulder, while many children with BBPP have internal rotation of approximate $40^{\circ}$. It has been shown that there is an increase in net joint force at the shoulder in flexion and extension as the internal rotation position of the shoulder increases, which is hypothesized as an etiology for joint malformation [10].

\section{Treatment}

Consistent therapy exercises are recommended for at least 6 months after diagnosis and for several years for those with severe palsy and/or surgery. It is recommended that older children have exercises to build up the involved, weak muscles and for all children to have range of motion (ROM) exercises [11]. Splints or orthoses are used in infants and children with BPP, both from the first weeks of life as well as postoperatively. These are for positioning to minimize contractures and to improve biomechanics to maximize function of the arm [12•].

One study of 319 children showed serial casting decreases elbow flexion contracture by an average of $30 \%$ but that splinting did not significantly improve the elbow ROM [13]. A smaller study of 19 patients found that serial casting and splinting for elbow flexion contracture were both effective in improving contracture; $53 \%$ of their patients were noncompliant with treatment and lost range of motion during their noncompliant period [14].

Electrical stimulation on a daily basis has been shown to improve muscle size and function even in denervated muscle [15]. Recommendations have been made for starting electrical stimulation as soon as possible after injury, multiple times a day [12•]. Biofeedback has been described as useful for treatment of abnormal co-contraction and for joint position $[12 \bullet, 16]$. Botulinum toxin $\mathrm{A}$ has also been described as useful in this situation $[11,17,18]$. It has been reported that one injection is often sufficient [11]. Botulinum toxin has been used in combination with therapies, serial casting and constraint induced movement therapy $[12 \bullet, 17]$.

In a study 35 infants with posterior subluxation and dislocation of the shoulder were treated with botulinum toxin $\mathrm{A}$ at $\sim 5$ months of age with resultant reduction of the shoulder joint. This was maintained over time, which reduced the need for surgical procedures in the majority of these infants. There were no complications noted with use of the toxin [19].

\section{Function}

Hand function can be severely impaired even in children with upper plexus injuries. A study of 25 children showed those with good or excellent shoulder function had hand function examination results $80 \%$ of that of the uninvolved hand. They were less accurate and took longer time to complete the tasks with the difference significantly worse than the difference for the dominant versus nondominant hand in the general population [20]. The importance of shoulder external rotation in ADLs was corroborated by a study that limited this motion in unaffected volunteers by use of a custom shoulder orthosis that limited shoulder movement. There was perceived increased difficulty during all ADLs tested and higher self report of disability scores while wearing these external rotationlimiting orthoses [21].

Evaluating functional tests for children with BBPP showed that the penny and the button tests were good predictors of impairment, and the box and block test correlated with stereognosis [22].

Pediatric outcome data collection instrument (PODCI) scores for children with BBPP are lower than age-matched peers in both global scores and upper extremity function, showing a decrease in activity and participation components [23]. A study of 23 children who underwent shoulder external rotation tendon transfers compared preoperative to 1-year postoperative studies. This showed that active shoulder abduction improvement, but not passive ROM changes, correlated with PODCI scores for upper extremity function and global function [24].

One study of 85 children with BBPP showed $88 \%$ playing sports, which is similar to the general pediatric population. They also played similar types of sports and had similar types of sports injuries [25]. Similarly, in another long-term study of 70 children, over two thirds regularly participated in sports, with no significant relationship between ROM and participation [26]. 


\section{Complications/Secondary Effects}

An elbow flexion contracture greater than $10^{\circ}$ was observed in approximately half of children with BBPP at a median age of 5 years. One third of the children had a contracture over $30^{\circ}$, and another one third of those with an elbow flexion contracture had an additional upper extremity contracture. There was no significant association with the severity of brachial plexus involvement [13].

Changes in the arms of children with birth brachial plexus palsy have recently been described to continue for a longer time than was previously thought. Seventy children were followed from 5 years old to the teenage years and were found to have a slight deterioration of elbow function over this time period, with $90 \%$ overall showing an elbow deficit. Some of these showed improvement with use of the splint but not with surgery. They also commonly had restricted shoulder external rotation [26].

Bone mineral density is decreased in the affected arm of children with BBPP. The deficit is more severe with more severe paralysis. Weight-bearing exercises improve the deficit [27].

Torticollis is commonly noted in BBPP, with $43 \%$ at presentation, with recovery in $62 \%$ by 5 weeks and no correlation with severity of BBPP [28].

Two large long-term studies showed none of the children with BBPP developed scoliosis [11, 26]. Abnormal growth of the affected arm and lack of cortical integration add to muscle pathology in BBPP [11].

\section{Central Nervous System (CNS)}

Brain reorganization after brachial plexus palsy is a recent topic of intense investigation. An early small study showed no contralateral brain activation on functional magnetic resonance imaging (fMRI) in a paralyzed arm mentally performing a motor task after BBP. Then there was symmetrical cerebral activation on fMRI several years after treatment, which was interpreted as recovery of neural input and output [29]. A later study looked at this with more time points throughout the recovery. The first fMRI was done at $0-5$ months post injury, then follow up at 12 months, then 3 and 4 years post injury. They were to flex and extend the elbow, imagining the movement if they were unable to do it physically. They showed decreased activation in the contralateral sensory motor cortex at the first fMRI, which decreased even more by 1 year post injury. This was felt to be due to the lack of neural input and output. By the third fMRI, the activation was increased and even more so by the fourth MRI, except in those rare cases where there was no clinical recovery. This was felt to indicate brain reorganization associated with peripheral changes in injury and recovery [30॰].

Neuropathic pain is commonly reported in adults and older children after traumatic brachial plexus injuries. It is not generally described after birth brachial plexus injury $[11,12 \bullet, 31]$. It has been hypothesized that this is due to increased central nervous system plasticity and possibly cortical reorganization $[12 \bullet, 31]$. Excellent sensory recovery in BBPP has also been attributed to the same etiology $[12 \bullet, 31]$.

\section{Quality of Life (QOL)}

A pilot study on quality of life (QOL) showed that most teens and their parents reported good overall QOL despite residual impairments. Functional and aesthetic factors were responsible for most QOL differences. Understanding patient expectations and QOL can help guide care [32]. Medical decision-making for teens must be multifaceted with both patient- and system-dependent factors. Four key items described for the families were knowledge acquisition, multidisciplinary care, adolescent autonomy, and patient expectations and treatment desires. Parental decisions were heavily influenced by system-dependent factors [33].

The impact of birth brachial plexus injury on parents has recently been studied. Parents are moderately affected by this socially, personally and financially. The age of the child and roots involved were not found to make a difference in the degree of impact [34]. As this is clarified further, more specific social support can be given.

\section{Surgical Access}

It has been estimated that $4-34 \%$ of infants with BBPP have deficits requiring surgery. It was hypothesized that this is undertreated and that insurance status may have an impact on this. Surgical utilization rate increased from $1.1 \%$ in the early 1990 s to $3.2 \%$ in 2006 . Treatment varied significantly with insurance status, from $3.8 \%$ for those with private insurance, $2.9 \%$ for those with Medicaid and $0.7 \%$ for those who are self pay, supporting the concern about limited access to care for the uninsured and underinsured [35].

\section{Surgical Approach}

\section{Surgical Intervention}

The decision [36] to perform a surgical intervention for children with obstetrical brachial plexus injuries has been 
consistent throughout our experience with treating these patients. A protocol established at Texas Children's Hospital that followed the general recommendations of Gilbert [37] and other corroborating investigators at the time has been implemented at The Texas Brachial Plexus Institute with a few modifications. Our recommendations are to perform primary reconstructions when patients meet any of the following criteria: (1) the patient has total paralysis of the arm without improvement at 3 months of age; (2) the patient has no biceps function at 3-6 months of age; (3) the patient has no active movement or less than antigravity movement of the arm at 3-6 months of age. Patients presenting to our center after the 3-12-month window are evaluated and if they meet any of the above criteria are scheduled for surgery. Patients who present with obstetric injuries after 12-18 months of age are no longer candidates for primary reconstructions as the timing of nerve regeneration and motor end plate atrophy has lapsed [38]. These patients are then evaluated for secondary reconstructive procedures.

\section{Primary Operative Innovations}

The surgical approach at The Texas Brachial Plexus Institute is done by utilizing a two-microsurgeon team for initial dissection and exploration in addition to harvesting and grafting of nerves. This approach has facilitated the surgical procedure and made intraoperative nerve testing and grafting much more integrated and time efficient.

During the dissection, meticulous care is taken utilizing $5.5 \times$ magnification loupes and the use of the Checkpoint Stimulator to help identify all aspects of the brachial plexus and surrounding nerves safely. Initial dissection, identification and testing of the brachial plexus at Texas Children's Hospital previously required the use of a neurosurgeon and an electrophysiologist. We now utilize the Checkpoint stimulator to help delineate the plexus anatomy quickly and effectively, decreasing the operative time significantly. An electrophysiologist experienced in this area is rare and if available is recommended. If not, then this stimulator also assists in evaluating motor nerve and muscle function. An experienced electrophysiologist will properly assess proximal electrical continuity via somatosensory evoked potentials. If not available, then root ruptures versus root avulsions are determined grossly by inspection of the transected proximal nerve root. If the nerve root reveals good fascicular structure then grafting to the nerve root is done. However, if the nerve root lacks fascicular structure an avulsion is diagnosed and another root is utilized or an extraplexal neurotization is considered. If there is any doubt of viability to the nerve root, a frozen section of the questionable nerve root is sent to pathology to evaluate the suitability of the root for grafting.
The reconstructive strategy at our center has also continued the strategy established with our work at Texas Children's Hospital [39] with a much lower threshold for excision of a neuroma if the electrical testing does not elicit a significant muscular response. When a neuroma in continuity exists, a meticulous neurolysis is performed to all of the roots, trunks, divisions and branches. Following the neurolysis either autologous grafts are utilized to bypass the neuroma or a new nerve allograft is utilized taking care to ensure minimal graft length with tension-free coaptation during the bypass.

Until recently, the use of nerve autografts has been the gold standard for nerve grafting and reconstruction [40]. Numerous nerve conduits, both synthetic and autologous, have been utilized for reconstructions [41, 42]. However, none of these attempted conduits have proven as or more effective than a nerve autograft. A new nerve allograft [43, 44] has been introduced to the market that has allowed us to have an adjunct to nerve autografts for brachial plexus reconstructions. We have utilized these nerve allografts to bypass neuromas successfully in reconstruction cases with conducting neuromas. In addition, once the allografts and the autografts have been sutured into position, another product made from porcine small intestine submucosa, an axoguard [45], has been shown to decrease epineurial scarring, remodels into similar epineurium and facilitates revascularization around the surgically repaired brachial plexus. These nerve axoguards are wrapped around the roots, trunks, divisions and branches securing the brachial plexus and the nerve grafts. Patients then have seprafilm placed into the incisions to further minimize scarring, and wounds are then closed. Patients then undergo a 2-week recovery period in a splint followed by aggressive occupational or physical therapy (OT or PT).

Patients who are found to have nonconducting or poorly conducting neuromas follow our standard reconstructive strategy of excision of all of the neuroma and scar, with resection of the plexus back to normal fascicular anatomy at the root and trunk/branch level. Reconstruction of the plexus is then done keeping in mind appropriate donorrecipient combinations, utilizing one or both sural nerves as autografts. Nerve allografts are used to maximize the reconstruction of healthy nerve fascicles since the sural nerve autografts are frequently not sufficient to completely graft all of the plexus. These nerve allografts become more important when the distance for reconstruction exceeds 3-4 cm, thus requiring longer sural nerve grafts, which limited the total roots that we could previously graft. Now using both the allografts and the sural nerve grafts ensures that the entire diameter of the proximal root and its fascicles has contact with a directional conduit for regeneration. In addition, after the grafting is complete, the entire plexus is then covered with the axoguards to help minimize the 
loss of sprouting nerve buds and to minimize peripheral scarring that can compress the reconstructed plexus.

Results to date have shown favorable outcomes with our reconstructive methods. We have had no adverse effects or results in the utilization of the nerve allografts or nerve axoguards. The vast majority of patients presented with upper plexus involvement $\mathrm{C} 5, \mathrm{C} 6$ and $\mathrm{C} 7$ neuromas. Functional improvement has been seen in all patients, improving two muscle grades or more to have muscle activity against gravity. As has been previously seen by other colleagues such as Gilbert [46], there has been a subset of patients that have then required secondary procedures to correct residual deformities.

\section{Secondary Surgical Innovations}

We regularly monitor and evaluate our patients utilizing the Mallet score and the British Medical Research Council grading system. When patients reach a plateau in functional improvement or regress despite ongoing and aggressive OT and PT, on average about 18-24 months of age, secondary surgical procedures are then implemented depending on each individuals deficits. Previously at Texas Children's Hospital, most secondary procedures included the release of the subscapularis muscle from the scapular attachments to improve external rotation in addition to other surgical maneuvers. Patients were then splinted after surgery for a period of 4-6 weeks followed by OT and PT. Upon continued follow-up of these patients, some patients developed a "freezing" of the humerus and the scapula. Patients are able to abduct their shoulder almost to near normal range. However, these patients are then unable to completely adduct their arm as the scapula wings posteriorly and limits the complete adduction of the arm. These arms are also locked in an internally rotated position without the ability to actively or passively externally rotate despite aggressive and ongoing therapy.

As a result of these subsets of patients, current studies have been started to attempt to formulate a surgical plan with the assistance of a pediatric shoulder specialist to correct this scapulohumeral fusion. In addition, patients currently undergoing secondary surgical procedures no longer have the subscapularis released as previously done. These patients are then also splinted for a shorter period of 2 weeks constantly and then 2 weeks at night. From our initial findings, we have not seen any children develop this scapulohumeral fusion and have continued to demonstrate the success of shoulder abduction improvement previously seen at other centers such as those reported by Dr. Gilbert [46].

Additional attempts to correct defects of external rotation have been addressed by innovative methods of local muscle transfers such as the trapezius muscle [47] on either the ipsilateral or the contralateral side [48]. Initial results of such transfers have shown excellent results in patients that have not responded to more traditional muscle and tendon transfers. These patients have shown increased external rotation from zero to a mean of $20^{\circ}$ as reported by Dr. Elhassan et al. [49]. In patients who also lack shoulder abduction, the trapezius muscle has been transferred successfully to restore shoulder stability in addition to increasing shoulder abduction, either alone or with other muscle and tendon transfers of the shoulder [47]. The use of the trapezius muscle has proven very effective, and several recommendations exist for its use and inclusion in reconstructions of the shoulder. The unique nature of the muscle and its innervation out of the usual zones of injury have made this muscle very versatile.

\section{Conclusion}

Many advances in the evaluation and treatment of children with BBPP have recently been developed. The scientific knowledge of the impact of the nervous system, including the brain, with imaging and other sciences contributing, has been significant. There have been advances in conservative and surgical treatments. Functional and longer term outcome studies are shedding light on more results and will help direct even more specific care.

Acknowledgments We would like to thank Drs. Rita Lee, Saleh Shenaq and Jack Laurent for their dedication to the care of children with brachial plexus palsy and the development of this field until the end of their lives. With this part of their life's work, they impacted the lives of thousands of children and their families, as well as trainees and colleagues.

\section{Compliance with Ethics Guidelines}

Conflict of Interest M. R. Nelson declares no conflicts of interest. A. H. Amenta declares no conflicts of interest.

Human and Animal Rights and Informed Consent This article does not contain any studies with human or animal subjects performed by any of the authors.

\section{References}

Papers of particular interest, published recently, have been highlighted as:

- Of importance

1. Malessy MJA, Pondaag W, Yang LJS, et al. Severe obstetric brachial plexus palsies can be identified at 1 month of age. PLoS One. 2011; 6:e26193, 1-8. 
2. - Van Dijk JG, Pondaag W, Buitenhuis SM, Van Zwet EW, et al. Needle electromyography at 1 month predicts paralysis of elbow flexion at 3 months in obstetric brachial plexus lesions. Dev Med Child Neurol. 2012;54:753-758. New data on prediction of recovery by EMG.

3. Elvrum AG, Hulleberg G, Brandal M, et al. Long term prognosis of obstetric brachial plexus palsy. Dev Med Child Neurol. 2013;55:53.

4. Vanderhove KL, Bovid K, Alpert H, et al. Utility of electrodiagnostic testing and computed tomography myelography in the preoperative evaluation of neonatal brachial plexus palsy. J Neurosurg Pediatr. 2012;9:283-9.

5. Talbert RJ, Michaud LJ, Mehlman CT, et al. EMG and MRI are independently related to shoulder external rotation function in neonatal brachial plexus palsy. J Pediatr Orthop. 2011;31:194-204.

6. - Sheffler LC, Lattanza L, Sison-Williamson M, et al. Biceps brachii long head overactivity associated with elbow flexion contracture in brachial plexus birth palsy. J Bone Joint Surg Am. 2012;15:289-297. New information for etiology of contractures, opposing long held beliefs.

7. Suzuki O, Sunagawa T, Yokota K, et al. Use of quantitative intraoperative electrodiagnosis during partial ulnar nerve transfer to restore elbow flexion: the treament of eight patients following brachial plexus injury. J Bone Joint Surg Br. 2011;93:364-9.

8. Gosk L, Koszewicz M, Urban M, et al. Assessment of the prognostic value of horner syndrome in perinatal brachial plexus palsy. Neuropediatrics. 2011;42:4-6.

9. Russo SA, Kozin SH, Zlotolow DA, et al. Scapulothoracic and glenohumeral contributions to motion in children with brachial plexus palsy. J Shoulder Elbow Surg. 2013;23:327-38.

10. Kleiber T, Popovic N, Bahm J. A modeling approach to compute modification of net joint forces caused by coping movements in obstetric brachial plexus palsy. J Brachial Plex Periph Nerve Inj. 2013;8:10-8.

11. Bahm J, Ocampo-Pavez C, Disselhorst-Klug C, et al. Obstetric brachial plexus palsy. Dtsch Arztbel Int. 2009;106:83-90.

12. - Smania N, Berto G, La Marchina E, et al. Rehabilitation of brachial plexus injuries in adults and children. Eur J Phys Rehabil Med. 2012;48:483-506. Broad ranging information on rehabilitation.

13. Sheffler LC, Lattanza L, Hagar Y, et al. The prevalence, rate of progression, and treatment of elbow flexion contracture in children with brachial plexus birth palsy. J Bone Joint Surg Am. 2012;94:403-9.

14. Ho ES, Roy T, Stephens D, et al. Serial casting and splinting of elbow contractures in children with obstetric brachial plexus palsy. J Hand Surg Am. 2010;35:84-91.

15. Salmons S, Ashley Z, Sutherland H, et al. Functional electrical stimulation of denervated muscles: basic issues. Artif Organs. 2005;29:199-202.

16. Terzis JK, Kostopoulos VK. The surgical treament of brachial plexus injuries in adults. Plast Reconstr Surg. 2007;119:73e-92e.

17. Santamato A, Panza F, Ranieri M, et al. Effect of botulinum toxin type A and modified constraint induced movement therapy on motor function of upper limb in children with obstetrical brachial plexus palsy. Child Nerv Syst. 2011;27:2187-92.

18. Intiso D. Therapeutic use of botulinum toxin in neurorehabilitation. J Toxicol. 2012;2012:802893.

19. Ezaki M, Malungpaishrope K, Harrison RJ, et al. Onabotulinum toxin A injection as an adjunct in the treatment of pposterior shoulder subluxation in neonatal brachial plexus palsy. J Bone Joint Surg Am. 2010;92:2171-7.

20. Immermen I, Alfonso DT, Ramos LE, et al. Hand function in children with an upper brachial plexus birth injury: results of the nine-hole peg test. Dev Med Child Neurol. 2012;54:166-9.
21. Langer JS, Sueoka SS, Wang AA. The importance of shoulder external rotation in activities of daily living: improving outcomes in traumatic brachial plexus palsy. J Hand Surg Am. 2012;37:1430-6.

22. Mulcahey MJ, Kozin S, Merenda L, et al. Evaluation of the box and blocks test, steroegnosis and item banks of activity and upper extremity function in youths with brachial plexus birth palsy. J Pediatr Orthop. 2012;32:S114-22.

23. Bae DS, Waters PM, Zurakowski D. Correlation of pediatric outcomes data collection instrument with measures of active movement in children with brachial plexus birth palsy. J Pediatr Orthop. 2008;28:584-92.

24. Dedina RD, Bagley AM, Molitor F, et al. Comparison of pediatric outcomes data collection instrument scores and range of motion before and after tendon transfers for children with brachial plexus birth palsy. J Pediatr Orthop. 2008;28:259-64.

25. Dae DS, Zurakowski D, Avallone N, et al. Sports participation in selected children with brachial plexus birth palsy. J Pediatr Orthop. 2009;29:496-503.

26. Strombeck C, Krumlinde-Sundholm L, Remahl S, et al. Longterm follow-up of children with obstetric brachoal plexus palsy I: functional aspects. Dev Med Child Neurol. 2007;49:198-203.

27. Ibrahim IA, Hawamdeh ZM, Alsharif AA. Evaluation of bone mineral density in children with perinatal brachial plexus palsy: effectiveness of weight bearing and traditional exercises. Bone. 2011;49:499-505.

28. Hervy-Jumper SL, Justice D, Vanaman MM, et al. Torticollis associated with neonatal brachial plexus palsy. Pediatr Neurol. 2011;45:305-10.

29. Malessy MJA, Bakker D, Dekker J. Functional magnetic resonance imaging and control over the biceps muscle after intercostal-musculocutaneous nerve injury. J Neurosurg. 2003;98:261-8.

30. - Yoshikawa T, Hayashi N, Tajiri Y, et al. Brain reorganization in patients with brachial plexus injury: A longitudinal functional MRI study. Scientific World Journal. 2012;2012:501751. Data on brain response to brachial plexus palsy and response to treatment.

31. Anand P, Birch R. Restoration of sensory function and lack of long-term chronic pain syndromes after brachial plexus injury in human neonates. Brain. 2002;125:113-22.

32. Siquteri L, Larson BP, Chang KW, et al. Understanding quality of life and patient expectations among adolescents with neonatal brachial plexus palsy: a qualitative and quantitative pilot study. J Hnad Surg Am. 2013;38:2387-97.

33. Squiteri L, Larson BP, Chang KW, et al. Medical decisionmaking among adolescents with neonatal brachial plexus palsy and their families: a qualitative study. Plast Reconstr Surg. 2013;131:880e-7e.

34. Firat T, Oskay D, Akel BS, et al. Impact of obstetrical brachial plexus injury on parents. Pediatr Int. 2012;54:881-4.

35. Siquiteri L, Steggerda J, Yang LJ, et al. A national study to evaluate trends in the utilization of nerve reconstruction for treament of neonatal brachial plexus palsy. Plast Reconstr Surg. 2011;127:277-83.

36. Shenaq SM, Kim YS, Armenta AH, Nath RK, Cheng E, Jedrysiak A. The surgical treatment of obstetrical brachial plexus palsy. Plast Reconstr Surg. 2004;113(4):54E-67E.

37. Gilbert A. Long-term evaluation of brachial plexus surgery in obstetrical palsy. Hand Clin. 1995;11(4):583-94 (discussion 594-595).

38. Shenaq SM, Berzin E, Lee R, Laurent JP, Nath R, Nelson MR. Brachial plexus birth injuries and current management. Clin Plast Surg. 1998;25(4):527-36.

39. Shenaq SM, Armenta AH, Roth FS, Lee RT, Laurent JP. Current management of obstetrical brachial plexus injuries at Texas 
Children's Hospital Brachial Plexus Center and Baylor College of Medicine. Sem Plas Surg. 2005;19(1):42-55.

40. Griffin JW, Hogan MV, Chhabra AB, et al. Peripheral nerve repair and reconstruction. J Bone Joint Surg Am. 2013;95(23): 2144-51.

41. Deal DN, Griffin JW, Hogan MV, et al. Nerve conduits for nerve repair or reconstruction. J Am Acad Orthop Surg. 2012;20(2): 63-8.

42. Ducoc I, Fu R, Iorio M, et al. Innovative treatment of peripheral nerve injuries: combined reconstructive concepts. Ann Plast Surg. 2012;68(2):180-7.

43. Brooks DN, Weber RV, Chao JD, et al. Processed nerve allografts for peripheral nerve reconstruction: a multicenter study of utilization and outcomes in sensory, mixed, and motor nerve reconstructions. Microsurgery. 2012;32(1):1-14.

44. Cho MS, Rinker BD, Weber RV, et al. Functional outcome following nerve repair in the upper extremity using processed nerve allograft. J Hand Surg. 2012;37(11):2340-9.
45. Issacs J. Clinical experience with Axoguard ${ }^{\circledR}$ nerve connector as a coaptation aid for cable grafting. White Paper.

46. Gilbert A, Hentz VR, Tassin JL. Brachial plexus reconstruction in obstetrical palsy: Operative indications and postoperative results. In: Urbaniak JR, editor. Microsurgery for major limb reconstruction. St. Louis: Mosby; 1987. p. 348.

47. Abdelaziz TA, Samir S, Magdy W. Restoring shoulder abduction in children with Erb's palsy: when to add trapezius transfer to a teres major transfer. J Bone Joint Surg Br. 2012;94(11):1579-82.

48. Elhassan B, Bishop AT, Hatcher RU, et al. Tendon transfer options about the shoulder in patients with brachial plexus injury. J Bone Joint Surg Am. 2012;94(15):1391-8.

49. Satbhai NG, Doi K, Hatori Y, et al. Contralateral lower trapezius transfer for restoration of shoulder external rotation in traumatic brachial plexus palsy: a preliminary report and literature review. J Hand Surg Eur. 2014;8:231. 